\title{
Correspondence
}

To the Editors:

\section{Should the Sri Lankan Japanese encephalitis vaccination schedule change as per WHO guideline?}

Sri Lanka Journal of Child Health, 2006; 35: 41-2

The foreign travellers to Sri Lanka are increasingly requesting the internationally recognized WHO schedule for Japanese Encephalitis (JE) vaccination which is days 0,7 and 30, rather than the Sri Lankan schedule of days 0,14 and 365 . The basis for this request is the evidence given in the WHO position paper on JE vaccination in their Weekly Epidemiological Record of $30^{\text {th }}$ October 1998.

"The mouse brain-derived JE vaccine (Nakayama strain or Beijing-1 strain) is given subcutaneously in doses of 0.5 or $1.0 \mathrm{ml}$, the lower dose being for children aged 1-3. The manufacturers of the internationally marketed vaccines recommend that primary immunization involves 2 injections at an interval of 1-2 weeks.

Many Asian countries have adopted a schedule of 2 primary doses, approximately 2-4 weeks apart, followed by a booster dose after 1 year with subsequent boosters at 3 yearly intervals for those living in endemic areas.

Immunogenicity studies conducted in western countries, where interference with other flaviviruses is unlikely, has shown that seroconversion was obtained only in about $80 \%$ of the vaccines following the primary 2 dose schedule. Furthermore, in $90 \%$ of the vaccines the titre of neutralizing antibody declined within 6-12 months to levels below the established protective titre level. However, in United States soldiers, a 3 dose schedule for primary immunization on days 0,7 and 30 resulted in $100 \%$ seroconversion, significantly higher titres of neutralizing antibodies and persistence of those high levels for at least 3 years.

\section{WHO position on JE vaccines}

Only a freeze dried, mouse brain-derived vaccine based on either Nakayama or Beijing strain of the JE vaccine is commercially available internationally. This vaccine is manufactured according to current international quality requirements and is in general considered efficient and safe for immunization of children.
Rare, but serious, neurological side-effects attributed to this JE vaccine have been reported from endemic as well as non-endemic regions. Furthermore, about $0.6 \%$ of Western adults, who in recent years were vaccinated prior to visits to endemic areas, have experienced allergic reactions to components of this vaccine. Therefore, an increased awareness of neurological as well as allergic adverse effects is necessary in endemic areas, in particular in countries where changing epidemiological patterns motivate JE vaccination of adults as well as children.

Although both genotypic and phenotypic variations of the JE viruses have been demonstrated, there is little evidence to suggest that the Nakayama vaccine strain results in reduced protection against infection by local virus strains.

Further information is needed concerning the duration of protection induced by JE vaccination. It is not known whether exposure of vaccinated individuals to natural infection contributes to protection. The possible interaction between JE virus infection and other flaviviruses prevailing in the region concerned requires further study. At least in theory, interactions between related flaviviruses could have an impact both on the result of primary immunization and on the requirement for boosters".

Reference: WHO. Weekly Epidemiological Record, 1998; 73: 337-44. Available from: http://www.who.int/wer

Priyanthi Molligoda

Consultant Paediatrician, Apollo Hospitals, Colombo 
